Published in final edited form as:

Science. 2013 September 13; 341(6151): . doi:10.1126/science.1235226.

\title{
Circulating Tumor Cells
}

\author{
Vicki Plaks, Charlotte D. Koopman, and Zena Werb \\ Department of Anatomy, University of California-San Francisco, San Francisco, CA 94143-0452, \\ USA
}

Zena Werb: zena.werb@ucsf.edu

\section{Abstract}

Much remains to be learned about CTCs and their clinical potential as biomarkers and therapeutic targets.

The major cause of cancer-associated mortality is tumor metastasis, but our understanding of this process is far from complete. During successful dissemination, tumor cells invade the surrounding tissue of the primary tumor, intravasate into blood and lymphatic vessels, translocate to distant tissues, extravasate, adapt to the new microenvironment, and eventually seed, proliferate, and colonize to form metastases. Because dissemination mostly occurs through the blood, circulating tumor cells (CTCs) that have been shed into the vasculature and may be on their way to potential metastatic sites are of obvious interest (1). Here we discuss what is known about CTCs, and suggest future research directions that may help realize their clinical potential.

The presence of CTCs in cancer patients was first detected in 1869 (2). Numerous studies in the past decade have shown that CTCs may be used as a marker to predict disease progression and survival in metastatic (2-4) and possibly even in early-stage cancer patients (5). High CTC numbers correlate with aggressive disease, increased metastasis, and decreased time to relapse (1). Moreover, CTCs can initiate metastasis in a xenograft model (6). If CTCs are indeed a source of metastatic cells, they could become an integral part of tumor staging criteria, which are currently focused on the primary tumor. Because blood collection is simple and minimally invasive, CTCs could be used as a real-time marker for disease progression and survival. CTCs also have the potential to guide therapeutic management (7), indicate therapy effectiveness or necessity, even in the absence of detectable metastases, and offer insights into mechanisms of drug resistance. They could be used as a surrogate endpoint marker in clinical trials (4), but could also become a treatment target (7). Despite this great potential, the use of CTCs faces many hurdles (see the figure).

CTCs have a diameter that is three to four times as large as the bores of capillaries in distant organs, so they would be expected to become trapped there $(1,8)$. That CTCs can be detected in the blood implies that only extremely small and/or plastic CTCs can keep circulating. And because some CTCs circulate as microclusters (9), these would likely also lodge in capillaries. Thus, it is unclear whether actual "circulating" tumor cells are indeed the source of metastases. Based on these points, it would be logical to assume that variations exist in the spatial and temporal distributions of CTCs within the circulation. Indeed, most solid malignancies have typical patterns of metastasis according to the localization of the primary tumor, suggesting at least partial filtration of CTCs. Thus, the site of blood collection for CTC detection may be critical. If CTCs need to be collected from various sites according to cancer type, blood sampling may not be as minimally invasive. Adding to this is the current requirement of large quantities of blood for CTC isolation and detection. Extracting blood from vessels draining the tumor may thus not be feasible and calls for the development of CTC detection devices that can handle small sample volumes. However, this 
poses an additional problem, because CTCs are extremely rare (7). For example, only $1.43 \%$ of patients with progressive breast cancer had $>500$ CTCs per $7.5 \mathrm{ml}$ of blood (6).

Moreover, lymphatic spread is still poorly understood (8), but could also be a route for tumor cell dissemination. CTCs may also be difficult to detect because they can become cloaked by platelets or by coagulation factors, thereby shielding them from the immune system. Indeed, high platelet count (thrombocytosis) is associated with poor prognosis in many cancers. Thus, a potentially metastatic subpopulation of CTCs may be currently undetectable.

Not all CTCs may be clinically relevant. Patients with various benign inflammatory colon diseases also harbored viable circulating epithelial cells detected by current CTC assays, whereas healthy subjects did not (10). Additionally, in a mouse model of pancreatic cancer, CTCs were found in the blood before the appearance of a primary tumor (5). Thus, current CTC assays are limited in distinguishing between cancer cells, noncancerous tumor components, and benign cells.

CTCs may be shed from different locations within tumors, which are heterogeneous in nature, and even from metastases. Indeed, frequently there is a clear discrepancy in gene expression between primary tumors and CTCs, as well as heterogeneity within the CTC population $(7,11,12)$. It may become possible to identify the tissue of origin of CTCs by using expression profiling to detect organ-specific metastatic signatures. This would help to localize small metastatic lesions and to guide further diagnostic and therapeutic strategies.

Cancer-associated traits in some cancers have been traced to so-called cancer stem cells (CSCs). The traits that define CSCs, i.e., self-renewal, tumor-initiating, motile, invasive, and heightened resistance to apoptosis, are also instrumental for metastasis $(1,13)$, implying that, in cancers that follow the cancer stem cell model, CTCs with high metastatic potential might be CSCs.

There is an ongoing discussion on whether tumor cells undergo epithelial-mesenchymal transition (EMT) during dissemination, resulting in a more mesenchymal or even more stem cell-like phenotype. However, current CTC detection methods mostly use the epithelial marker epithelial cell adhesion molecule (EpCAM), which may under-estimate CTC number and potentially miss a critical subpopulation $(1,7)$. Although it has yet to be shown in spontaneously arising cancers in vivo, EMT can induce non-CSCs to enter a CSC-like state, generating CSCs de novo $(1,13)$. However, the role of EMT in enabling metastatic dissemination is yet to be fully proven and may only operate in a fraction of cancer cells that are in close contact with adjacent reactive stroma (1). Present at the tumor edges, these cells may dislodge and become blood borne, i.e., CTCs. If only CTCs that undergo EMT are those with self-renewal capability and proliferative potential essential for macroscopic metastases (13), markers for metastatic CTCs should include EMT markers. Indeed, in many tumors, carcinoma cells exhibit a partial mesenchymal state $(1,9)$, a phenotype absent from normal tissues (1). Still, it remains to be determined what fraction of CTCs lose some or all EpCAM expression and undergo (partial) EMT and whether these (or any) CTCs have increased metastatic seeding potential or heightened resistance to systemic therapy and therefore greater prognostic value. It is also unknown whether tumorigenic and nontumorigenic cells differ in their capacity to circulate in cancers that follow the cancer stem cell model. Thus, the overall relationship between CSCs and CTCs remains unclear.

That some CTCs are undetectable and not all detected CTCs have metastatic potential indicates that CTC enumeration is not a good marker for disease staging and prognosis. Instead, it is instrumental to design biomarkers based on the gene sets and genomic profile of CTC subsets that predict homing and colonization to specific distant metastatic sites or 
even sites of primary tumor origin. Advanced CTC analysis is being made possible by constant technical improvements in CTC detection and isolation (7), although there are still unresolved issues, specifically the need to standardize detection assays (12).

The development of single-cell analyses that can detect gene expression in individual CTCs has revealed interesting data, such as the finding of human epidermal growth factor receptor 2 (HER2)-positive CTCs in HER2-negative breast cancers (11). Such studies have expedited clinical trials on CTCs and indeed, the numerous ongoing trials are indicative of the vast interest in these cells. Recent genomic analysis of CTCs has detected singlenucleotide and copy-number variations generally accepted as driving forces in cancer (14). However, CTC genomics is still in its infancy, mainly due to a lack of technologies capable of isolating sufficient numbers of CTCs to analyze somatic mutations $(14,15)$, and the lack of suitable material with which to compare results due to CTC heterogeneity.

The potential clinical value of CTCs is clear: Early detection and treatment of metastatic spread are key for disease outcome, and CTCs offer the ability to target metastasis in real time. Although CTCs are not yet proven to be the metastatic cells, there is no evidence that they are incapable of being so. However, a simple enumeration of CTCs without molecular characterization may lead to wrongful clinical assumptions and consequences. Elucidating CTC biology will also help standardize detection and isolation of the potentially metastatic subpopulation of CTCs. The next frontier in the CTC field is their characterization using the constantly improving single-cell "omics" techniques (16). This will ultimately determine the clinical value of CTCs as biomarkers and therapeutic targets.

\section{Acknowledgments}

This work was supported by funds from the NIH (R01 CA057621 and U01 ES019458 to Z.W.) and by a Department of Defense postdoctoral fellowship to V.P. (W81XWH-11-01-0139).

\section{References and Notes}

1. Chaffer CL, Weinberg RA. Science. 2011; 331:1559. [PubMed: 21436443]

2. Cristofanilli M, et al. N. Engl. J. Med. 2004; 351:781. [PubMed: 15317891]

3. Cohen SJ, et al. J. Clin. Oncol. 2008; 26:3213. [PubMed: 18591556]

4. de Bono JS, et al. Clin. Cancer Res. 2008; 14:6302. [PubMed: 18829513]

5. Rhim AD, et al. Cell. 2012; 148:349. [PubMed: 22265420]

6. Baccelli I, et al. Nat. Biotechnol. 2013; 31:539. [PubMed: 23609047]

7. Alix-Panabières C, Pantel K. Clin. Chem. 2013; 59:110. [PubMed: 23014601]

8. Chambers AF, Groom AC, MacDonald IC. Nat. Rev. Cancer. 2002; 2:563. [PubMed: 12154349]

9. Yu M, et al. Science. 2013; 339:580. [PubMed: 23372014]

10. Pantel K, et al. Clin. Chem. 2012; 58:936. [PubMed: 22205690]

11. Hayashi N, et al. Int. J. Clin. Oncol. 2012; 17:96. [PubMed: 21671160]

12. Strati A, Kasimir-Bauer S, Markou A, Parisi C, Lianidou ES. Breast Cancer Res. 2013; 15:R20. [PubMed: 23497487]

13. Mani SA, et al. Cell. 2008; 133:704. [PubMed: 18485877]

14. Zong C, Lu S, Chapman AR, Xie XS. Science. 2012; 338:1622. [PubMed: 23258894]

15. Heitzer E, et al. Cancer Res. 2013; 73:2965. [PubMed: 23471846]

16. Wang D, Bodovitz S. Trends Biotechnol. 2010; 28:281. [PubMed: 20434785] 


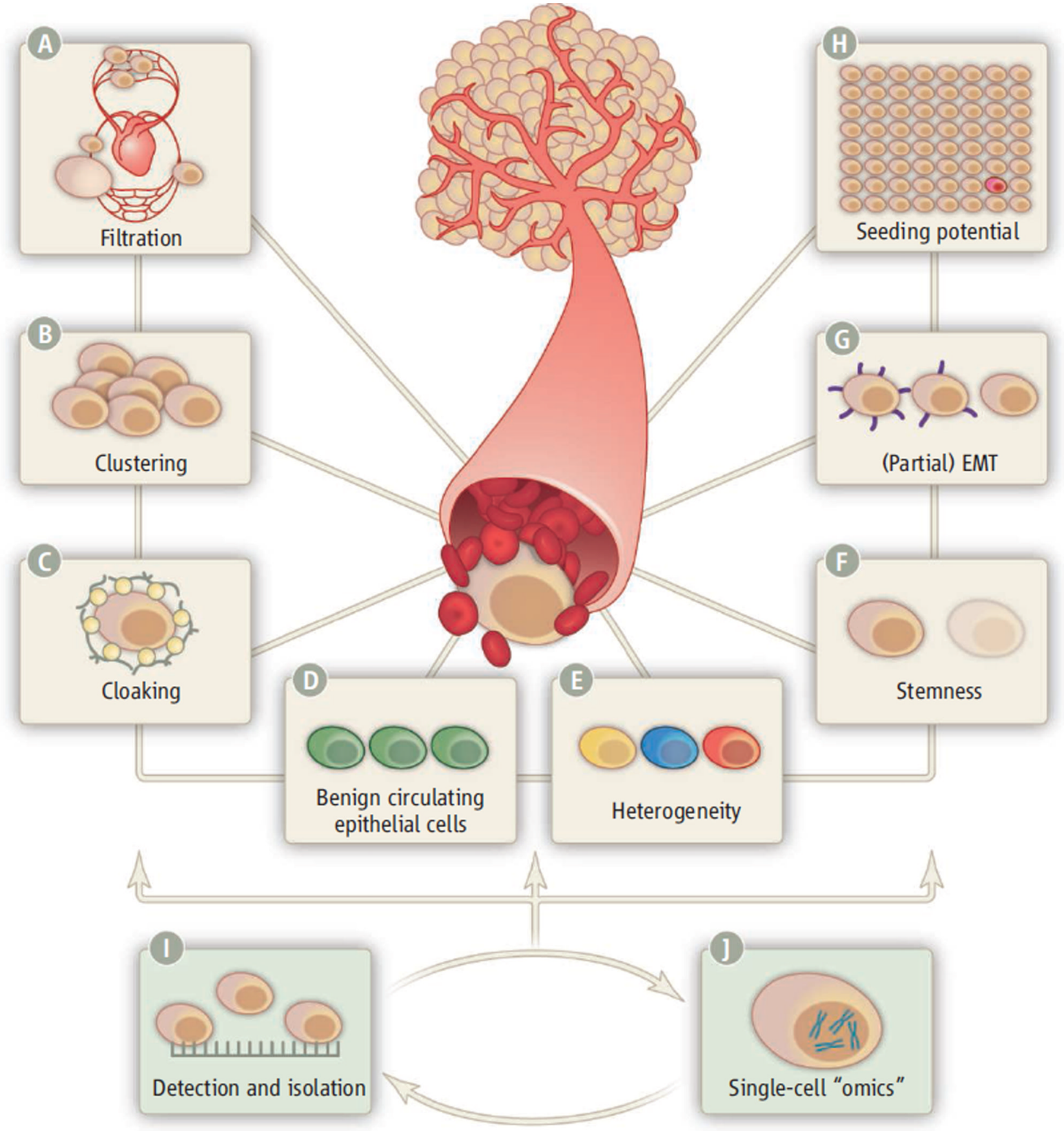

1.

Hurdles and solutions in CTC research. Biophysical factors that may diminish the detection of CTCs include (A) filtration of large CTCs in smaller capillaries, (B) clustering of tumor cells that lodge in capillaries, and (C) cloaking of CTCs by platelets or coagulation factors. Biological factors that likely complicate the detection and isolation of clinically relevant populations of CTCs that currently rely on epithelial markers include (D) the presence of benign circulating epithelial cells, $(\mathbf{E})$ the large heterogeneity among CTCs, $(\mathbf{F})$ the possible stemness of a subpopulation of CTCs, $(\mathbf{G})$ the (partial) epithelial-mesenchymal transition (EMT) that some CTCs undergo during dissemination, and $(\mathbf{H})$ the unclear 
seeding potential of detected CTCs. Future research should use technologies focused on (I) improving the detection and isolation of CTCs, and (J) single-cell "omics." 